\title{
RETENCIÓN URINARIA TRANSITORIA EN EL REIMPLANTE URETERAL EXTRAVESICAL BILATERAL SIMULTÁNEO
}

Miguel Castellán, Manuel García Mérida y Rafael Gosálbez.

Division of Pediatric Urology. Miami Children Hospital and Jackson Memorial Hospital. Department of Urology.

University of Miami. Miami. Florida. USA.

\begin{abstract}
Resumen.- OBJETIVO: Se realiza un estudio retrospectivo a los pacientes sometidos a reimplante ureteral bilateral extravesical simultáneo y se reportan los resultados y la incidencia de retención urinaria transitoria.
\end{abstract}

MÉTODOS: Se evalúan a 127 pacientes con reflujo vesicoureteral primario a los que se les realizó reimplante ureteral bilateral simultáneo en un período de 8 años. El sexo fue femenino en $92(72,4 \%)$ y masculino en $35(27,6 \%)$. En todos los pacientes, al terminar el procedimiento, se dejó un catéter uretral tipo Foley en veiiga entre 24 y 72 horas. Postoperatoriamente se evaluaron con ultrasonidos del tracto urinario en el primer mes y con cistograma miccional en el tercero.
RESULTADOS: El seguimiento postoperatorio medio fue de 4.01 años. No hubo ningún caso de obstrucción ureteral. El cistograma postoperatorio demostró la resolución del reflujo vesicoureteral bilateral en 122 (96\%) pacientes y persistencia del mismo en 5 (4\%). Al retirar la sonda vesical postoperatoria $7 / 127$ (5.5\%) pacientes presentaron retención urinaria. Se han dividido los pacientes en dos grandes grupos basados en el tiempo y en las variantes técnicas quirúrgicas empleadas. El primero (junio de 1998 a enero de 2001) lo constituyen 57 reimplantes extravesicales bilaterales con 5 (8.7\%) casos de retención urinaria transitoria, comenzando a miccionar de forma normal a las 24 horas, 48 horas, 72 horas, 5 días y 4 semanas cada uno de los niños. El segundo grupo (febrero de 2001 a Octubre de 2006) lo forman 70 reimplantes extravesicales bilaterales y hubo solo 2 (2.85\%) casos de retención urinaria transitoria, comenzando a orinar los dos de forma normal a las 24 horas.

CONCLUSIONES: El reimplante vesicoureteral extravesical bilateral es un procedimiento con un elevado índice de éxito y una mínima morbilidad. Puede ocasionar disfunción miccional retencionista transitoria. En nuestra serie la utilización de cauteterización bipolar y una disección más limitada y cuidadosa del uréter distal, disminuyó la incidencia de retención urinaria transitoria postoperatoria.

Palabras clave: Reimplante. Reflujo vesicoureteral. Extravesical. Retención urinaria.

Summary.- OBJECTIVES: We report the outcome and incidence of urinary retention after bilateral extravesical reimplant in patients with primary vesicoureteral reflux.

METHODS: We retrospectively evaluated the chart of $127 \mathrm{pa-}$ tients, 92 females and 35 males, who underwent correction of primary vesicoureteral reflux using the extravesical approach. Mean patient age at surgery was 3.93 years. Postoperatively the urethral catheter was removed after 24 to 72 hours and 
a voiding trial was done. Surgical outcomes were analyzed specifically for perioperative complications and resolution of reflux on postoperative VCUG.

RESULTS: Mean follow-up was 4.01 years. Postoperative VCUG showed resolution of reflux in 122 (96\%) patients. Urinary retention developed in $7 / 127$ patients (5.5\%). In 57 patients in whom the surgery was done from 06-1998 to 01 2001 , urinary retention developed in $5 / 57(8.7 \%)$ for 1 day (1), 2 days (1), 5 days (2) and 4 weeks (1). In 70 patients in whom the surgery was done from 02-2001 to 10-2006, urinary retention developed in $2 / 70$ (2.85\%) for 1 day (2).

CONCLUSIONS: Bilateral extravesical vesicoureteral reimplant can be associated with temporary urinary retention. In the last 5 years, with a careful and limited dissection close to the distal ureter and used of bipolar cautery when necessary, we were able to decreased the risk of postoperative urinary retention.

Keywords: Vesicoureteral reimplant. Extravesical. Urinary retention. Vesicoureteral reflux.

\section{INTRODUCCIÓN}

El reimplante ureteral extravesical es un procedimiento que se realiza desde hace muchos años para el tratamiento del reflujo vesicoureteral con excelentes resultados a corto y largo plazo. Sin embargo, varios autores han advertido de la posibilidad de retención miccional transitoria después de realizar el procedimiento extravesical de forma bilateral y simultánea (1-3). El índice de retención urinaria tras la reimplantación bilateral oscila entre 0-26\% (1-6). Desde que Lich y Gregoire popularizaron este procedimiento se han descrito varias modificaciones técnicas y se han realizado diversos estudios anatómicos con el fin de disminuir la morbilidad e incrementar el éxito de la operación $(7,8)$.

En este trabajo se realiza un estudio retrospectivo de los pacientes sometidos a reimplante ureteral bilateral extravesical simultáneo y se reportan los resultados y la incidencia de retención urinaria transitoria. Se analizan también los cambios quirúrgicos efectuados en los últimos 5 años y que ocasionaron una disminución importante del porcentaje de retención urinaria transitoria.

\section{MATERIALES Y MÉTODOS}

Desde junio de 1998 a octubre de 2006 se realiza un estudio retrospectivo de 127 pacientes con reflujo vesicoureteral primario a los que se les realizó un reimplante ureteral extravesical bilateral simultáneo. El sexo fue femenino en $92(72,4 \%)$ y masculino en $35(27,6 \%)$. La edad media a la cirugía fue de 3.93 años $(4$ meses - 24 años). Las indicaciones de la cirugía fueron infección urinaria a pesar de tratamiento médico, persistencia o no resolución del reflujo a pesar de tratamiento médico, deterioro de la función renal y no cumplimiento por parte de los pacientes o padres con el tratamiento médico. Los grados de reflujo preoperatorio se han dividido en dos grandes grupos, reflujos bajo grado (grados I a III), y reflujos de alto grado (grados IV y V). El de bajo grado lo constituyen 186 $(73,2 \%)$ unidades renales y el de alto grado $68(26,8 \%)$. Tres pacientes tenían antecedentes de tratamiento bilateral previo con inyección subureteral de dextranómero del ácido hialurónico sin resultado.

El reimplante vesicoureteral se realizó en todos los casos según la técnica de Lich-Gregoire en $Y$ invertida sin desinserción del uréter. La técnica quirúrgica utilizada en nuestro servicio fue publicada anteriormente en mayor detalle (García Mérida y cols.) (9). En nuestra publicación previa se evaluaron retrospectivamente los pacientes en los que la cirugía fue realizada desde junio de 1998 hasta enero de 2001. En los últimos cinco años hemos realizado cambios en la técnica quirúrgica, especialmente en los reimplantes extravesicales bilaterales. Los cambios son:

1) Una disección más limitada y cuidadosa del uréter distal, que realizamos sólo hasta el cruce de los vasos tributarios de la vesical inferior, evitando la incisión en $Y$ invertida en los casos bilaterales y las disecciones amplias de la unión vesicoureteral;

2) La disección del detrusor sólo se realiza con las tijeras de Metzenbaum;

3) La cauterización, sí es necesaria, sólo se efectúa con pinzas bipolares;

4) La arteria umbilical obliterada sólo se liga y secciona en casos limitados, evitando hacerlo de forma sistemática. Después de suturar la herida operatoria ésta se infiltró con solución de bupivacaina al $0.25 \%$. En ningún caso se utilizó anestesia caudal. En todos los pacientes, al terminar el procedimiento, se dejó un catéter uretral tipo Foley en la vejiga entre 24 y 72 horas. La analgesia postoperatoria fue realizada con codeína asociada a paracetamol y con morfina en los no respondedores al tratamiento previo. No se administró oxibutinina en el postoperatotio temprano. Se estimuló a todos los pacientes para que deambularan pronto y para que ingirieran líquidos y tuvieran una dieta normal en el menor tiempo postoperatorio posible. Al retirar la sonda se mide el volumen urinario evacuado y se realiza una exploración física para descartar que estén en retención urinaria. En casos de duda se evalúa el residuo postmiccional ya sea por sondaje vesical o por ultrasonido vesical. Postoperatoriamente todos los pacientes se evaluaron con ultrasonidos del tracto urinario en el primer mes y con cistograma miccional isotópico en el tercero. Las historias clínicas fueron evaluadas retrospectivamente poniendo énfasis especial en el éxito quirúrgico de la operación y en los pacientes con retención miccional postoperatoria.

\section{RESULTADOS}

El seguimiento postoperatorio medio fue de 4.01 años. No hubo ningún caso de obstrucción ureteral inmediata ni tardía. El cistograma miccional postoperatorio demostró la resolución del reflujo vesicoureteral bilateral 
en $122(96 \%)$ y 5 pacientes $(4 \%)$ presentaron reflujo vesicoureteral persistente. En 2 pacientes $(1,6 \%)$ el reflujo fue de menor grado que el previo y fueron sometidos a profilaxis antibiótica; la evolución fue a la curación espontánea en los siguientes 15 meses de postoperatorio. Con estos resultados el índice de éxito con una sola operación es del $97.6 \%$ de los pacientes. Otros 2 pacientes con reflujo vesicoureteral persistente, uno bilateral y del mismo grado y otro unilateral de menor grado, se les realizó una inyección subureteral de dextranómero del ácido hialurónico con buenos resultados postoperatorios. El paciente restante presentó un reflujo vesicoureteral unilateral de menor grado y se encuentra actualmente bajo tratamiento con profilaxis antibiótica.

Al retirar la sonda vesical, entre las 24 a 72 horas postoperatorias, $7 / 127(5.5 \%)$ pacientes presentaron retención urinaria. Se han dividido los pacientes en dos grandes grupos basados en el tiempo y en las variantes técnicas quirúrgicas empleadas:

1) El primero (junio de 1998 a enero de 2001) lo constituyen 57 reimplantes extravesicales bilaterales con $5(8.7 \%)$ casos de retención urinaria transitoria. Dos de estos pacientes, uno de 7 años de sexo femenino y otro de 4 años de sexo masculino, ambos con reflujo grado III bilateral, comenzaron a orinar en forma espontánea a las 24 y 48 horas respectivamente, necesitando entre medio de sondajes intermitentes. Los otros 3 pacientes, con reflujo III de un lado y IV del otro, necesitaron recolocación de la sonda vesical por ascenso de la creatinina plasmática. A 2 pacientes, uno de 7 años de sexo femenino y otro de 1 año de sexo masculino, se les dejó la sonda durante 3 días a ambos y luego comenzaron a orinar en forma normal. A otro paciente de sexo femenino de 5 años de edad se le retiró la sonda vesical a los 5 días y preciso de sondajes intermitentes por elevados residuos postmiccionales por el término de 4 semanas y se curó después.

2) El segundo grupo (febrero de 2001 a Octubre de 2006) lo forman 70 reimplantes extravesicales bilaterales y hubo solo $2(2.85 \%)$ casos de retención urinaria transitoria. Ambos pacientes fueron de sexo femenino $(2$ y 6 años respectivamente) y la sonda vesical se recolocó por 24 horas y luego ambos comenzaron a orinar espontáneamente.

\section{DISCUSIÓN}

El abordaje extravesical para la corrección del reflujo vesicoureteral es utilizado con éxito desde hace mucho tiempo. La técnica original fue desarrollada por Dodson (10) en perros y publicada en humanos por Lich y cols. (7) y Gregoir (8), quien extendió la disección distal al orificio ureteral. La obstrucción ureteral es una complicación que rara vez se produce en el postoperatorio del reimplante extravesical.

En nuestra serie no hubo ningún caso de obstrucción ureteral secundaria a la cirugía, en forma similar a las series de Lipski (1), Barrieras (3) y Minevich (11). El éxito del reimplante extravesical supera el $95 \%$ de los pa- cientes en diferentes series. En nuestra serie, un $96 \%$ de los pacientes presentaron curación del reflujo vesicoureteral bilateral en la cistografía postoperatoria. Dos pacientes que presentaron un reflujo persistente de menor grado que el original y que fueron sometidos a profilaxis antibiótica tuvieron una curación espontánea del mismo en los siguientes 15 meses postoperatorios, llevando el índice de éxito con una sola operación a $97.6 \%$ de los pacientes.

La retención urinaria transitoria postoperatoria es una complicación conocida del reimplante extravesical bilateral realizado de forma simultánea por lo que muchos cirujanos no la utilizan. El índice reportado de retención urinaria tras un reimplante ureteral extravesical bilateral varia entre 0-26\% (1-6). Desde la publicación original de la técnica extravesical se han publicado diversas modificaciones técnicas con el fin de disminuir el índice de retención urinaria temporal en el tratamiento del reflujo bilateral. El detrusor a nivel del trígono y la base de la vejiga tiene áreas muy ricas en inervacion.

Leissner y cols. (12) reportaron un estudio en cadáveres humanos que revelo que la porción principal del plexo pélvico se sitúa alrededor de $1.5 \mathrm{~cm}$. dorsal y medial a la unión ureterovesical. Ramas del plexo pélvico terminan en el uréter distal, trígono y recto. Durante la realización de un reimplante vesicoureteral en cadáveres reportaron un alto riesgo de daño al plexo pélvico y sus ramas eferentes. David y cols. (5) publicaron su serie de reimplante extravesical bilateral utilizando los conceptos de Leissner y cols. (12) y reportaron un índice de retención urinaria de $2 \%$. En su trabajo recomiendan limitar la disección medial del uréter, realizándola mayoritariamente en la cara lateral del uréter.

En 2005, McAchran y cols. (4) publicaron su serie de reimplante extravesical bilateral y en 50 pacientes no reportaron ningún caso de retención urinaria transitoria. Además de limitar la disección medial al uréter distal también recomiendan evitar una amplia disección del uréter en proximidad a la arteria umbilical obliterada, ya que según el autor se afectan las fibras aferentes del plexo pélvico. Muchos autores reconocen diferentes factores que en el periodo postoperatorio temprano pueden contribuir a disminuir el índice de retención urinaria como son: evitar la sobredistensión vesical realizando micciones períodicas, deambular pronto, evitar anticolinérgicos y no utilizar analgésicos narcóticos.

En nuestra serie el índice de retención urinaria tras el reimplante extravesical bilateral fue de 5.5\% (7/127) pacientes. Entre junio de 1998 a enero de 2001 un $8.7 \%$ (5/57) de los pacientes presentaron retención urinaria transitoria. Desde esta fecha hasta octubre de 2006 sólo un $2.85 \%(2 / 70)$ de los pacientes tuvieron un episodio de retención urinaria por un tiempo mucho más limitado (24 horas). Nosotros creemos que la disminución del índice de retención urinaria en nuestra serie en los últimos 5 años se debe en gran medida a los cambios realizados en la técnica original, principalmente a una disección menos amplia del uréter distal, a la utilización en forma limitada de la cauterización y al uso de la pinza bipolar, hechos que disminuyen el daño a las ramas del plexo pélvico. 


\section{CONCLUSIONES}

El reimplante vesicoureteral extravesical bilateral es un procedimiento con un elevado índice de éxito y una mínima morbilidad. Puede ocasionar disfunción miccional retencionista transitoria y es recomendable evaluar en forma reglada el habito miccional de los pacientes en el postoperatorio inmediato. En nuestra serie, en los últimos 5 años, con la utilización de cauteterización bipolar y una disección más limitada y cuidadosa del uréter distal, disminuyó la incidencia de retención urinaria postoperatoria.

\section{BIBLIOGRAFÍA Y LECTURAS RECOMENDADAS (*lectura de interés $\mathrm{y}^{* *}$ lectura fundamental)}

*1. LIPSKI, B.A.; MITCHELL, M.E.; BURNS, M.W.: "Voiding dysfunction after bilateral extravesical ureteral reimplantation". J. Urol., 159: 1019, 1998.

2. FUNG, L.C.; McLORIE, G.A.; JAIN, U. y cols.: "Voiding efficiency after ureteral reimplantation: a comparison of extravesical and intravesical techniques". J. Urol., 153: 1972, 1995.

*3. BARRIERAS, D.; LAPOINTE, S.; REDDY, P. y cols.: "Urinary retention after bilateral extravesical ureteral reimplantation: Does dissection distal to the ureteral orifice have a role?". J. Urol., 162: 1197, 1999.
4. McACHRAN, S.E.; PALMER, J.S. J.: "Bilateral extravesical ureteral reimplantation in toilet trained children: Is 1-day hospitalization without urinary retention possible?". Urol., 174: 1991, 2005.

5. DAVID, S.; KELLY, C.; POPPAS, D.: "Nerve sparing extravesical repair of bilateral vesicoureteral reflux: Description of technique and evaluation or urinary retention”. J. Urol., 172: 1617, 2004.

6. MAROTTE, J.B.; PRESTON SMITH, D.: "Extravesical ureteral reimplantations for the correction of primary reflux can be done as outpatient procedures". J. Urol., 165: 2228, 2001.

7. LICH, R.J.; HOWERTON, L.; DAVIS, L.A.: "Recurrent urosepsis in Children”. J. Urol., 86: 554, 1961.

8. GREGOIR, W.: "Le traitement chirurgical du reflux vesico-ureteral congenital”. Acta Chir. Belg., 63: 432, 1964.

9. GARCIA MERIDA, M.; GOSALBEZ, R.; RIUS DIAZ, F. y cols.: "Reflujo vesicoureteral esencial y reimplantacion ureteral extravesical en niños". Actas Urol. Esp., 30: 602, 2006.

10. DODSON, A.I.: "Some improvements in the technique of ureteroneocystostomy". J. Urol., 55: 225, 1964.

*11. MINEVICH, E.; ARONOFF, D.; WACKSMAN, J. y cols.: "Voiding dysfunction after extravesical detrusorrhapy". J. Urol., 160: 1004, 1998.

**12. LEISSNER, J.; ALLHOFF, P.; WOLFF, W. y cols.: "The pelvic plexus and antireflux surgery: Topographical findings and clinical consequences". J. Urol., 165: 1652, 2001. 